tumor present after surgery that represents the most important prognostic factors. ESGO developed a list of quality indicators with an update in 2020 for certification of centers to ensure the best surgery for patients. These criteria include 24 complete surgeries per year in advanced stage III and IV ovarian cancer over the last 3 years. The main target is to ensure a complete resection rate of minimum require $>50 \%$ to an optimal > 65\%.

Methodology From 2018 the Department of Gynecology Oncology Center of Treviso Regional Hospital is trying to obtain the Esgo accreditation for the ovarian cancer.

Result(s)* Analyzing all the cases (from 2018 to 2020) we report a total of 66 high grade ovarian cancer, a complete resection rate of $73 \%$, with a rate of primary debulking surgeries of $74 \% ; 83 \%$ of the surgeries were performed by a certified gynecologic oncologist. The decision for any major therapeutic intervention has been taken by a multidisciplinary team in the $80 \%$ of cases. More than $95 \%$ patients had a treatment planned at a multidisciplinary team meeting (including a surgical specialist, a radiologist, a pathologist and a medical oncologist), more than $95 \%$ patients had a required pre operative workup. More than $90 \%$ of patients had a minimum required elements in pathology reports, we recorded prospectively all of the post operative complications. According to all of these aspects our center obtained a score of 32 points.

Conclusion* Actually the quality of the team work in our center has improved in the last three years. We started in 2018 with 18 cases of ovarian cancer moving up in the last two years to 24 cases. In 2020 we reported a lower rate of complete resection due to the more complex surgery for a more radical surgery to get a complete cytoreduction. We realized that since we begin to collect data for the accreditation we improved our surgical approach for the advance ovarian cancer.

\section{OVARIAN CANCER ONSET ACROSS DIFFERENT BRCA MUTATION TYPE}

${ }^{1} \mathrm{C}$ Marchetti*, 'M D'indinosante, ${ }^{2} \mathrm{~A}$ Minucci, ${ }^{1} \mathrm{~L}$ Giacò, ${ }^{3} \mathrm{C}$ Cassani, ${ }^{3} \mathrm{G}$ La Cava, ${ }^{1} \mathrm{R}$ Ergasti, ${ }^{1} \mathrm{G}$ Scambia, ${ }^{1} \mathrm{~A}$ Fagotti. ${ }^{1}$ Agostino Gemelli University Policlinic, Department of Woman and Child Health and Public Health, Roma, Italy; ${ }^{2}$ Agostino Gemelli University Policlinic, Molecular and Genomic Diagnostics Uni, Roma, Italy; ${ }^{3}$ Fondazione I.R.C.C.S. Policlinico San Matteo, Pavia, Italy

\subsection{6/ijgc-2021-ESG0.461}

Introduction/Background* Mutations in BRCA-genes have been identified as predisposing to hereditary breast and ovarian cancers. Little is known about how ovarian cancer risks differs across different BRCA mutation type. The purpose of our study is to identify the correlation between specific type of BRCA mutations and (1) age of onset of highgrade serous ovarian cancer (HGSOC) and (2) patients' survival.

Methodology Retrospective multicentric series of newly diagnosed HGSOC-patients with FIGO Stage III-IV, assessed for germline (g)BRCA status. BRCA gene mutations were classified into 5 groups: deletion, insertion, no-sense, missense and splicing.

Result(s)* A total of 214 patients were included in the analysis. $143(67.1 \%)$ had a gBRCA1-pathogenic variant (PV) and $71(33.1 \%)$ had a gBRCA2-PV.
Abstract 747 Table 1 Distribution of BRCA mutation and age of onset

\begin{tabular}{|l|l|l|l|l|l|l|}
\hline & $\begin{array}{l}\text { All cases } \\
\mathrm{Nr}(\%)\end{array}$ & $\begin{array}{l}\text { Age } \\
\text { Mean (SD) }\end{array}$ & $\begin{array}{l}\text { BRCA-1 } \\
\mathbf{N r}(\%)\end{array}$ & $\begin{array}{l}\text { Age } \\
\text { Mean (SD) }\end{array}$ & $\begin{array}{l}\text { BRCA-2 } \\
\mathbf{N r}(\%)\end{array}$ & $\begin{array}{l}\text { Age } \\
\text { Mean (SD) }\end{array}$ \\
\hline All cases & $\mathbf{2 1 4}$ & $\mathbf{5 4 . 7 5 ( 1 0 . 9 )}$ & $\mathbf{1 4 3}$ & $\mathbf{5 3 ( 1 0 . 9 )}$ & $\mathbf{7 1}$ & $\mathbf{5 7 . 2 ( 1 0 . 5 )}$ \\
\hline Deletion & $93(43.5)$ & $55(10.4)$ & $55(38.5)$ & $53.4(10.1)$ & $38(53.5)$ & $57.2(10.7)$ \\
\hline Insertion & $40(18.7)$ & $58(11.1)$ & $26(18.2)$ & $57.7(11.7)$ & $14(19.7)$ & $59.6(10.1)$ \\
\hline No sense & $39(18.2)$ & $49.7(9.0)$ & $30(21.0)$ & $48.8(9.7)$ & $9(12.7)$ & $53.0(5.5)$ \\
\hline Missense & $32(15)$ & $55.4(12.9)$ & $25(17.5)$ & $54.6(12.6)$ & $7(9.9)$ & $58.2(14.9)$ \\
\hline Splicing & $10(4.7)$ & $55.3(8.9)$ & $7(4.9)$ & $54.5(7.6)$ & $3(4.2)$ & $57.0(13.4)$ \\
\hline
\end{tabular}

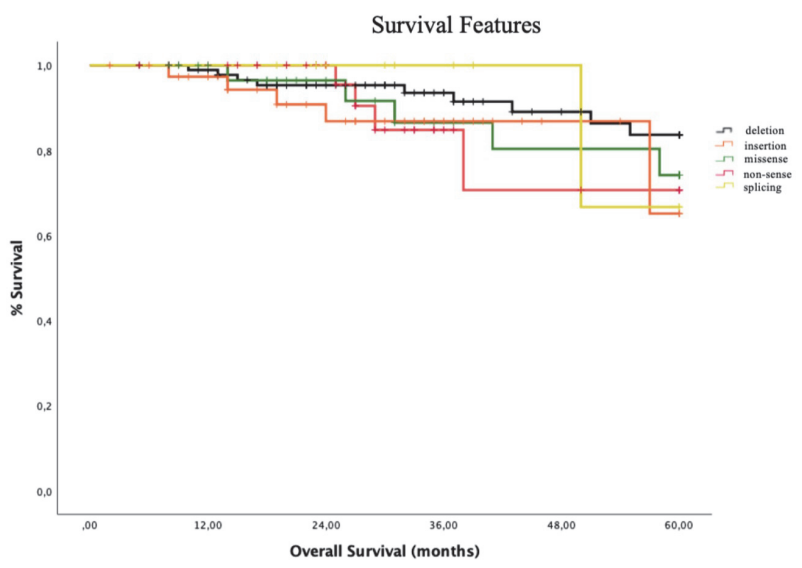

Abstract 747 Figure 1 overall survival in patients with different type of BRCA mutations

Overall, the mean age of onset was 54.75 years (10.9 SD) with a difference of around 4 years between patients having BRCA 1 and BRCA2 mutations (53yrs, SD 10.9 vs. 57.2yrs, SD $10.5 ; \mathrm{p}=0.018)$. The most frequent mutation found was deletion (42.9\%). Patients with no-sense mutation (18.2\%) had the youngest age of onset, both in BRCA1 and BRCA2 subgroups, with an earlier occurrence of around 6 and 4 years respectively (BRCA1 group: 48.8yrs, SD 9.7 vs.54.7yrs, SD10.9; $\mathrm{p}=0.008$ ) (BRCA2 group 53.0yrs SD 5.5 vs. 57.9yrs SD 10.9; $\mathrm{p}=0.04)$. Women with insertion $(18.7 \%)$ had the oldest age of onset, both in BRCA 1 (57.7yrs, SD 11.7 vs. 52.2yrs, SD10.6; $\mathrm{p}=0.028$ ) and BRCA2 (59.6yrs SD 10.1 vs. 56.6yrs SD 10.6) subgroups ( $\mathrm{p}=\mathrm{ns}$ ) (table 1$)$. No statistically significant difference in overall survival was found among the 5 groups examined (figure. 1).

Conclusion* Our study highlights for the first time that different types of BRCA mutations could indicate a different age for OC onset. If confirmed in larger series, it might have a relevant clinical impact, leading to a more tailored approach for risk-reducing surgery strategies for OC prevention. Moreover, as we initially include only advanced stages in our analysis, further investigation on the time of onset of early BRCA mutated OC is currently ongoing.

\section{CORRELATION BETWEEN TUMOR MARKERS AND TUMOR BURDEN IN ADVANCED EPITHELIAL OVARIAN CANCER}

AG Glickman*, B Gil Ibanez, P Paredes, A Niñerola, M Tormo, N Carreras, N Agustí, M Del Pino, B Diaz-Feijoo, P Fusté, J Pahisa, A Torne. Hospital Clínic Barcelona

\subsection{6/ijgc-2021-ESGO.462}

Introduction/Background* The aim of this study was to correlate the serum concentrations of tumor markers HE4 and 